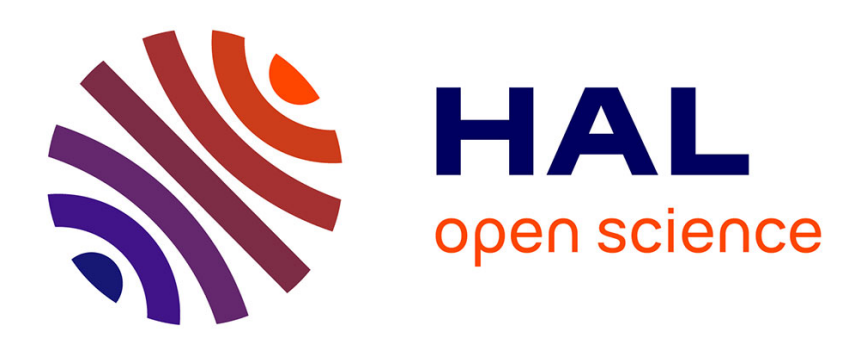

\title{
Shakedown theorems and asymptotic behaviour of solids in non-smooth mechanics
}

Michaël Peigney

\section{To cite this version:}

Michaël Peigney. Shakedown theorems and asymptotic behaviour of solids in non-smooth mechanics. EUROMECH - Colloquium 548 - Direct and variational methods for nonsmooth problems in mechanics, Jun 2013, Amboise, France. 7p. hal-00839292

\section{HAL Id: hal-00839292 \\ https://hal-enpc.archives-ouvertes.fr/hal-00839292}

Submitted on 27 Jun 2013

HAL is a multi-disciplinary open access archive for the deposit and dissemination of scientific research documents, whether they are published or not. The documents may come from teaching and research institutions in France or abroad, or from public or private research centers.
L'archive ouverte pluridisciplinaire HAL, est destinée au dépôt et à la diffusion de documents scientifiques de niveau recherche, publiés ou non, émanant des établissements d'enseignement et de recherche français ou étrangers, des laboratoires publics ou privés. 


\title{
Shakedown theorems and asymptotic behaviour of solids in non-smooth mechanics
}

\author{
Michaël Peigney \\ Université Paris-Est, Laboratoire Navier \\ (Ecole des Ponts ParisTech, IFSTTAR, CNRS), \\ F-77455 Marne-la-Valle Cedex 2, France \\ michael.peigney@ifsttar.fr
}

\begin{abstract}
Non-smooth mechanics is concerned with systems for which constraints are imposed on the physical quantities or their time-derivatives. This paper addresses the long-time behaviour of such systems when they are submitted to a given loading history. A motivation for this work is the study of shape-memory alloys structures, which are a typical example of systems for which an analysis in non-smooth mechanics is required. Extending the approach introduced by Koiter in plasticity, we state sufficient conditions for the energy dissipation to remain bounded in time, independently on the initial state. In the case of cyclic loadings, we also show that the long-time behaviour of systems in non-smooth mechanics is fundamentally different - and actually more complex - than in plasticity.
\end{abstract}

\section{Introduction}

This paper is concerned with the long-time behaviour of inelastic structures under prescribed loading histories. Much is known for elastic perfectly plastic structures: one of the earliest and most seminal contribution in that field has been made by Koiter [1], following a pioneering idea of Melan [2]. The so-called Melan-Koiter static theorem gives a sufficient condition for the energy dissipation to remain bounded with respect to time. That situation is refered to as shakedown, and is associated with the intuitive idea that the structure behaves elastically for time $t$ sufficiently large. The Melan-Koiter theorem has the distinctive property of being path-independent, i.e. independent on the initial state of the structure. In the particular case of cyclic loadings, it it also known [3,4] that the stress response $\sigma(t)$ always converges towards a cyclic response $\sigma_{\infty}(t)$ as $t \longrightarrow+\infty$. Similarly, the rate of plastic strain $\dot{\boldsymbol{\alpha}}(t)$ converges towards a cyclic response $\dot{\boldsymbol{\alpha}}_{\infty}(t)$. Moreover, both $\sigma_{\infty}(t)$ and $\dot{\boldsymbol{\alpha}}_{\infty}(t)$ have the same time period $T$ as the applied loading. The plastic strain $\boldsymbol{\alpha}(t)$ does not necessarily converge towards a cyclic response, since $\int_{0}^{T} \dot{\boldsymbol{\alpha}}_{\infty}(t) d t$ may be different from 0 . That situation is refered to as ratchetting and implies the collapse of the structure through the accumulation of plastic strain. In the case where $\int_{0}^{T} \dot{\boldsymbol{\alpha}}_{\infty}(t) d t=0$, one classically distinguishes the cases of shakedown $\left(\dot{\boldsymbol{\alpha}}_{\infty}=0\right)$ and alternating plasticity $\left(\dot{\boldsymbol{\alpha}}_{\infty} \neq 0\right)$. In that last case, the plastic strain $\boldsymbol{\alpha}(t)$ converges towards a cyclic but non constant response $\boldsymbol{\alpha}_{\infty}(t)$. A crucial property of elastic perfectly plastic structures is that the asymptotic rate of plastic strains $\dot{\boldsymbol{\alpha}}_{\infty}$ is unique. This implies that the asymptotic regime (shakedown, alternating plasticity, or ratchetting) is path-independent. That property has fostered the development of direct methods aiming EUROMECH - Colloquium 548 Direct and variational methods for nonsmooth problems in mechanics Amboise, France $24-26$ June 2013 
at determining the asymptotic regime for a given cyclic loading, without using a step-by-step incremental analysis $[5,6,7,8,9]$.

All the results mentioned so far apply for elastic perfectly plastic structures, and can be directly extended to the $C$-class of generalized standard materials [10]. Outside of that framework, a lot of progress still remains to be made. Several attempts have been made to extend the Melan-Koiter theorem to various types of nonlinear behaviour (see [11] for an extensive review). However, as discussed in detail by Pham [11], some of the extensions proposed in the litterature lead to non path-independent results which are therefore of little practical use. This is notably the case for shape memory alloys: shakedown in shape-memory alloys structures has recently been studied in [12], but the shakedown theorem obtained by those authors has latter been recognized not to be path-independent [11].

Shape Memory Alloys (SMAs) display peculiar properties such as the superelastic behaviour or the shape memory effect, which are both the result of a solid/solid phase transformation between different crystallographic structures (known as austenite and martensite). Much effort has been devoted to developing constitutive laws for describing the behaviour of SMAs. The phase transformation is typically described by an internal variable $\alpha$ which - depending on the complexity of the material model - may be scalar or vectorial. A fundamental observation is that the internal variable $\alpha$ must comply with some a priori inequalities, resulting from the mass conservation in the phase transformation process. As a consequence, the internal variable $\alpha$ is constrained to take values in a set $\mathcal{T}$ that is not a vectorial space. The presence of such constraints constitutes a crucial difference with plasticity models, and calls for special attention when the structural evolution problem is considered. This last point has been noted by Govindjee and Miehe [13] in the context of numerical methods for simulating SMA structures: apart from few exceptions $[13,14]$, most existing numerical methods handle the constraints in an ad hoc fashion, for lack of a consistent formulation of the time continuous evolution problem. It has to be observed, however, that mathematically consistent models of evolution problems in shapememory alloys have been proposed $[15,16]$. One possible approach is to resort to the so-called "non-smooth mechanics" framework (see [15] and references therein), which is not restricted to shape-memory alloys and actually applies in the general situation where constraints are physically imposed on the state variables or their time-derivative. This paper is devoted to studying the asymptotic behaviour of solids in such a framework.

\section{Constitutive laws}

The local state of the material is described by the strain $\boldsymbol{\epsilon}$ and an internal variable $\boldsymbol{\alpha}$, living respectively in vectorial spaces denoted by $\mathbb{E}$ and $\mathbb{A}$. The scalar products in $\mathbb{A}$ and $\mathbb{E}$ are denoted by . and :, respectively. The associated norms are denoted by || and \|\| , i.e. $|\alpha|=\sqrt{\boldsymbol{\alpha} . \boldsymbol{\alpha}}$ for any $\boldsymbol{\alpha} \in \mathbb{A}$ and $\|\boldsymbol{\epsilon}\|=\sqrt{\boldsymbol{\epsilon}: \boldsymbol{\epsilon}}$ for any $\boldsymbol{\epsilon} \in \mathbb{E}$. We assume that the variable $\boldsymbol{\alpha}$ is constrained to take values in a convex and closed subset $\mathcal{T}$ of $\mathbb{A}$. Adopting the framework of generalized standard materials in non-smooth mechanics $[10,15]$, the behaviour of the material is determined by the free energy function $w: \mathbb{E} \times \mathbb{A} \mapsto \mathbb{R}$ and the dissipation potential $\Phi: \mathbb{A} \mapsto \mathbb{R}$. More precisely, denoting by $\dot{\alpha}$ the left-time derivative of $\boldsymbol{\alpha}$, the constitutive equations are

$$
\begin{gathered}
\boldsymbol{\sigma}=\frac{\partial w}{\partial \boldsymbol{\epsilon}}(\boldsymbol{\epsilon}, \boldsymbol{\alpha}), \boldsymbol{A}=-\frac{\partial w}{\partial \boldsymbol{\alpha}}(\boldsymbol{\epsilon}, \boldsymbol{\alpha}) \\
\boldsymbol{A}=\boldsymbol{A}^{d}+\boldsymbol{A}^{r}, \boldsymbol{A}^{d} \in \partial \Phi(\dot{\boldsymbol{\alpha}}), \boldsymbol{A}^{r} \in \partial I_{\mathcal{T}}(\boldsymbol{\alpha})
\end{gathered}
$$


where $\sigma$ is the stress, $\boldsymbol{A}$ is the thermodynamical force associated to $\alpha$, and $\partial$ denotes the subdifferential operator [17]. We consider free energy functions $w(\boldsymbol{\epsilon}, \boldsymbol{\alpha})$ of the form

$$
w(\boldsymbol{\epsilon}, \boldsymbol{\alpha})=\frac{1}{2}(\boldsymbol{\epsilon}-\boldsymbol{K} . \boldsymbol{\alpha}): \boldsymbol{L}:(\boldsymbol{\epsilon}-\boldsymbol{K} . \boldsymbol{\alpha})+f(\boldsymbol{\alpha})
$$

where $\boldsymbol{L}: \mathbb{E} \mapsto \mathbb{E}$ is a symmetric positive linear mapping, $\boldsymbol{K}: \mathbb{A} \mapsto \mathbb{E}$ is a linear mapping, and $f: \mathbb{A} \mapsto \mathbb{R}$ is a positive differentiable function (not necessarily linear). The dissipation potential $\Phi$ is assumed to satisfy the following standard properties:

$$
\begin{aligned}
& \text { (i) } \Phi \text { is convex, positive, null at the origin } \\
& \text { (ii) } \exists r>0 \text { such that }\{\boldsymbol{A} \in \mathbb{A}|| \boldsymbol{A} \mid \leq r\} \subset \partial \Phi(0)
\end{aligned}
$$

With the form (3) of the free energy, the relation (1) becomes

$$
\boldsymbol{\sigma}=\boldsymbol{L}:(\boldsymbol{\epsilon}-\boldsymbol{K} . \boldsymbol{\alpha}), \boldsymbol{A}={ }^{t} \boldsymbol{K}: \boldsymbol{\sigma}-f^{\prime}(\boldsymbol{\alpha})
$$

where ${ }^{t} \boldsymbol{K}: \mathbb{E} \mapsto \mathbb{A}$ is defined by $\boldsymbol{\alpha} .\left({ }^{t} \boldsymbol{K}: \boldsymbol{\sigma}\right)=\boldsymbol{\sigma}:(\boldsymbol{K} . \boldsymbol{\alpha})$ for all $(\boldsymbol{\alpha}, \boldsymbol{\sigma}) \in \mathbb{A} \times \mathbb{E}$. The relation (5) shows that the total strain $\epsilon$ is the sum of an elastic strain $L^{-1}: \sigma$ and an inelastic strain $K . \alpha$. The most common plasticity models fall in the format (3)-(4). So does a wide range of SMA models $[18,19,13,20,21,22,23]$. In models of shape memory alloys, the internal variable is constrained to lie in a bounded set. Coupling phase transformation with plasticity typically leads to situations in which the internal variable is required to lie in a unbounded set. In such models, the internal variable is indeed decomposed as $\boldsymbol{\alpha}=\left(\boldsymbol{\alpha}_{1}, \boldsymbol{\alpha}_{2}\right)$ where $\boldsymbol{\alpha}_{1} \in \mathbb{A}_{1}$ is the internal variable related to plasticity, and $\alpha_{2} \in \mathbb{A}_{2}$ is related to phase transformation. The plastic variable $\boldsymbol{\alpha}_{1}$ can take any value in $\mathbb{A}_{1}$ while $\boldsymbol{\alpha}_{2}$ must belong to a bounded subset $\mathcal{T}_{2}$ of $\mathbb{A}_{2}$. The internal variable $\alpha$ is thus constrained to take values in the unbounded set $\mathcal{T}=\mathbb{A}_{1} \times \mathcal{T}_{2}$. The free energy $w$ and the dissipation potential $\Phi$ have the following structure:

$$
\begin{array}{ll}
w\left(\boldsymbol{\epsilon}, \boldsymbol{\alpha}_{1}, \boldsymbol{\alpha}_{2}\right)= & \frac{1}{2}\left(\boldsymbol{\epsilon}-\boldsymbol{K}_{1} \cdot \boldsymbol{\alpha}_{1}-\boldsymbol{K}_{2} . \boldsymbol{\alpha}_{2}\right): \boldsymbol{L}:\left(\boldsymbol{\epsilon}-\boldsymbol{K}_{1} . \boldsymbol{\alpha}_{1}-\boldsymbol{K}_{2} . \boldsymbol{\alpha}_{2}\right) \\
& +f_{1}\left(\boldsymbol{\alpha}_{1}\right)+f_{2}\left(\boldsymbol{\alpha}_{2}\right) \\
\Phi\left(\dot{\boldsymbol{\alpha}_{1}}, \dot{\boldsymbol{\alpha}_{2}}\right)= & \Phi_{1}\left(\dot{\boldsymbol{\alpha}}_{1}\right)+\Phi_{2}\left(\dot{\boldsymbol{\alpha}}_{2}\right)
\end{array}
$$

where $\boldsymbol{K}_{1}: \mathbb{A}_{1} \mapsto \mathbb{E}$ and $\boldsymbol{K}_{2}: \mathbb{A}_{2} \mapsto \mathbb{E}$ are linear mappings, $f_{1}, f_{2}$ are positive functions, and $\Phi_{1}, \Phi_{2}$ both satisfy (4). The function $f_{1}$ (related to plastic variables) is convex, but $f_{2}$ (related to phase transformation variables) is not necessarily so. The dissipation $\Phi(\dot{\alpha})$ is the sum of a plastic dissipation $\Phi_{1}\left(\dot{\boldsymbol{\alpha}}_{1}\right)$ and a 'phase transformational' dissipation $\Phi_{2}\left(\dot{\boldsymbol{\alpha}}_{2}\right)$.

\section{Structural evolution problem}

We now consider the evolution of a structure submitted to a prescribed loading history. The constitutive material is assumed to satisfy the properties (3)-(4). The structure occupies a domain $\Omega$ and is submitted to body forces $\boldsymbol{f}^{d}$. Displacements $\boldsymbol{u}^{d}$ are imposed on a part $\Gamma_{u}$ of the boundary $\Gamma$, and tractions $\boldsymbol{T}^{d}$ are prescribed on $\Gamma_{T}=\Gamma-\Gamma_{u}$. The given functions $\boldsymbol{f}^{d}, \boldsymbol{u}^{d}, \boldsymbol{T}^{d}$ represent the loading of the structure. Those functions depend on position $x$ and time $t$, and the stress and state variables $(\sigma, \boldsymbol{\epsilon}, \boldsymbol{\alpha})$ in the structure are also expected to depend on $(\boldsymbol{x}, t)$. In order to alleviate the expressions, this dependence will be omitted in the notations, unless in the case of possible ambiguities. 
Quasi-static evolutions of the structure are governed by the following system:

$$
\begin{gathered}
\sigma \in \mathcal{K}_{\sigma}, \boldsymbol{\epsilon} \in \mathcal{K}_{\epsilon}, \boldsymbol{\alpha} \in \mathcal{T} \\
\boldsymbol{A}^{d} \in \partial \Phi(\dot{\boldsymbol{\alpha}}), \boldsymbol{A}^{r} \in \partial I_{\mathcal{T}}(\boldsymbol{\alpha}) \\
\boldsymbol{\sigma}=\boldsymbol{L}:(\boldsymbol{\epsilon}-\boldsymbol{K} . \boldsymbol{\alpha}) \\
{ }^{t} \boldsymbol{K}: \boldsymbol{\sigma}-f^{\prime}(\boldsymbol{\alpha})=\boldsymbol{A}^{d}+\boldsymbol{A}^{r}
\end{gathered}
$$

where $\mathcal{K}_{\sigma}$ and $\mathcal{K}_{\epsilon}$ are respectively the sets of admissible stress and strain fields, defined by $\mathcal{K}_{\sigma}=$ $\left\{\sigma \mid \operatorname{div} \boldsymbol{\sigma}+\boldsymbol{f}^{d}=0\right.$ in $\Omega ; \boldsymbol{\sigma} . \boldsymbol{n}=\boldsymbol{T}^{d}$ on $\left.\Gamma_{T}\right\}$ and $\mathcal{K}_{\epsilon}=\left\{\boldsymbol{\epsilon} \mid \boldsymbol{\epsilon}=\left(\nabla \boldsymbol{u}+{ }^{t} \nabla \boldsymbol{u}\right) / 2\right.$ in $\Omega ; \boldsymbol{u}=\boldsymbol{u}^{d}$ on $\left.\Gamma_{u}\right\}$. It is convenient to introduce the so-called fictitious elastic response $\left(\boldsymbol{\sigma}^{E}, \boldsymbol{\epsilon}^{E}\right)$ of the system, which is solution of

$$
\boldsymbol{\sigma}^{E} \in \mathcal{K}_{\sigma}, \boldsymbol{\epsilon}^{E} \in \mathcal{K}_{\epsilon}, \boldsymbol{\sigma}^{E}=\boldsymbol{L}: \boldsymbol{\epsilon}^{E}
$$

Setting $\boldsymbol{\rho}=\boldsymbol{\sigma}-\boldsymbol{\sigma}^{E}$ and noting that $\boldsymbol{\epsilon}=\boldsymbol{\epsilon}^{E}+\boldsymbol{L}^{-1}: \boldsymbol{\rho}+\boldsymbol{K} . \boldsymbol{\alpha}$, the system (7) can be recast as the evolution problem $\mathcal{P}\left(\sigma^{E}\right)$ defined as follows:

EVOLUTION PROBLEM $\mathcal{P}\left(\sigma^{E}\right)$.

$$
\begin{gathered}
\boldsymbol{\rho} \in \mathcal{K}_{\sigma}^{0}, \boldsymbol{\alpha} \in \mathcal{T} \\
\boldsymbol{A}^{d} \in \partial \Phi(\dot{\boldsymbol{\alpha}}), \boldsymbol{A}^{r} \in \partial I_{\mathcal{T}}(\boldsymbol{\alpha}) \\
\boldsymbol{L}^{-1}: \boldsymbol{\rho}+\boldsymbol{K} . \boldsymbol{\alpha} \in \mathcal{K}_{\epsilon}^{0} \\
{ }^{t} \boldsymbol{K}:\left(\boldsymbol{\sigma}^{E}+\boldsymbol{\rho}\right)-f^{\prime}(\boldsymbol{\alpha})=\boldsymbol{A}^{d}+\boldsymbol{A}^{r}
\end{gathered}
$$

The sets $\mathcal{K}_{\sigma}^{0}$ and $\mathcal{K}_{\epsilon}^{0}$ in (9) are defined by

$$
\begin{aligned}
& \mathcal{K}_{\sigma}^{0}=\left\{\boldsymbol{\sigma} \mid \operatorname{div} \boldsymbol{\sigma}=0 \text { in } \Omega ; \boldsymbol{\sigma} . \boldsymbol{n}=0 \text { on } \Gamma_{T}\right\} \\
& \mathcal{K}_{\epsilon}^{0}=\left\{\boldsymbol{\epsilon} \mid \boldsymbol{\epsilon}=\left(\nabla \boldsymbol{u}+{ }^{t} \nabla \boldsymbol{u}\right) / 2 \text { in } \Omega ; \boldsymbol{u}=0 \text { on } \Gamma_{u}\right\}
\end{aligned}
$$

\section{Shakedown theorems}

In this Section, we study the behaviour of solutions of $\mathcal{P}\left(\sigma^{E}\right)$ as time $t$ tends towards infinity. More precisely, we state conditions ensuring shakedown of the system, in the sense that the mechanical dissipation $\int_{0}^{t} \int_{\Omega} A^{d} . \dot{\alpha} d x d t$ remains bounded in time $t$. We refer to [24] for a detailed proof of those theorems.

Shakedown theorem 1 ( $f$ convex). If there exists $m>1, T \geq 0$ and time-independent fields $\boldsymbol{\rho}_{*} \in \mathcal{K}_{\sigma}^{0}, \boldsymbol{\alpha}_{*} \in \mathcal{T}, \boldsymbol{A}_{*}^{r} \in \partial I_{\mathcal{T}}\left(\boldsymbol{\alpha}_{*}\right)$ such that

$$
{ }^{t} \boldsymbol{K}:\left(m \boldsymbol{\sigma}^{E}(t)+\boldsymbol{\rho}_{*}\right)-f^{\prime}\left(\boldsymbol{\alpha}_{*}\right)-\boldsymbol{A}_{*}^{r} \in \partial \Phi(0) \text { for all } t>T
$$

then there is shakedown, whatever the initial condition is.

This result applies both if $\mathcal{T}$ is unbounded or bounded. In the particular case $\mathcal{T}=\mathbb{A}$, we have $\partial I_{\mathcal{T}}\left(\boldsymbol{\alpha}_{*}\right)=0$ and Theorem 1 recovers the shakedown condition given by Nguyen [25] for hardening plasticity. In the case where $\mathcal{T}$ is bounded, shakedown is actually ensured under less stringent conditions than those given in Theorem 1 . The following theorem can indeed be proved:

Shakedown theorem 2 ( $\mathcal{T}$ bounded). If there exists $m>1, T \geq 0$ and a time-independent field $\boldsymbol{A}_{*}^{r}$ such that

$$
m^{t} \boldsymbol{K}: \boldsymbol{\sigma}^{E}(t)-\boldsymbol{A}_{*}^{r} \in \partial \Phi(0) \text { for all } t>T
$$

then there is shakedown, whatever the initial condition is. 
It appears that the convexity of $f$ is not necessary for stating a shakedown theorem when $\mathcal{T}$ is bounded. This is a welcome feature for the shakedown analysis of SMA structures as the function $f$ associated with SMA models is not necessarily convex [20, 21].

Notice that the time-independent field $\boldsymbol{A}_{*}^{r}$ in Theorem 2 is free from any constraint. The shakedown condition in that theorem is thus only determined by the local amplitude of the elastic response $\sigma^{E}$. This situation is reminiscent of plasticity in linear kinematic hardening, for which shakedown is ensured under similar conditions [26].

Let us now consider coupled plasticity / phase transformation models of the form (6). For such models, the set $\mathcal{T}$ is unbounded and the function $f$ is not convex, so that neither Theorem 1 nor Theorem 2 applies. However, combining the reasoning leading to Theorems 1 and 2, the following can easily be proved:

Shakedown theorem 3 ( $f_{1}$ convex, $\mathcal{T}_{2}$ bounded). If there exists $m>1, T \geq 0$ and timeindependent fields $\boldsymbol{A}_{*}^{r} \in \mathbb{A}_{2}, \boldsymbol{\rho}_{*} \in \mathcal{K}_{\sigma}^{0}, \boldsymbol{\alpha}_{*} \in \mathbb{A}_{1}$ such that

$$
\begin{array}{ll}
{ }^{t} \boldsymbol{K}_{1}:\left(m \boldsymbol{\sigma}^{E}(t)+\boldsymbol{\rho}_{*}\right)-f_{1}^{\prime}\left(\boldsymbol{\alpha}_{*}\right) \in \partial \Phi_{1}(0) & \text { for all } t>T \\
m^{t} \boldsymbol{K}_{2}: \boldsymbol{\sigma}^{E}(t)-\boldsymbol{A}_{*}^{r} \in \partial \Phi_{2}(0) & \text { for all } t>T
\end{array}
$$

then there is shakedown, whatever the initial condition is.

\section{The three-bar problem}

In order to illustrate the results presented in section 4, we consider the three-bar problem represented on Figure 1. The three bars are identical and described by a model of the form (3)(4). A thermal strain $\epsilon_{T}$ is imposed in the middle bar. That strain $\epsilon_{T}$ is varying with time $t$ and takes values between 0 and a strictly positive value $\epsilon_{T}^{\max }$. The initial state $\left(\alpha_{1}, \alpha_{2}\right)(t=0)$ of the structure is taken in the form $\alpha_{1}(t=0)=\alpha_{2}(t=0)=\alpha^{0}$. In such conditions, the symmetry of the problem implies that the stress and strain in the lower and upper bars are equal at all time. The stress state of the structure can thus be represented by $\left(\sigma_{1}, \sigma_{2}\right)$ where $\sigma_{1}$ is the stress in the middle bar, and $\sigma_{2}$ is the stress in the two others. Similarly, the strain state of the structure is represented by $\left(\epsilon_{1}, \epsilon_{2}\right)$ where $\epsilon_{1}$ and $\epsilon_{2}$ are respectively the strain in the middle bar and in the two others. Analog notations are used for the internal variable $\alpha$. The constitutive laws in each bar is

$$
\begin{gathered}
\sigma_{1}=E\left(\epsilon_{1}-\alpha_{1}-\epsilon_{T}\right), \sigma_{2}=E\left(\epsilon_{2}-\alpha_{2}\right) \\
\boldsymbol{\alpha}_{i} \in \mathcal{T} \\
\sigma_{i}-f^{\prime}\left(\boldsymbol{\alpha}_{i}\right) \in \partial \Phi\left(\dot{\boldsymbol{\alpha}}_{i}\right)+\partial I_{\mathcal{T}}\left(\boldsymbol{\alpha}_{i}\right) \text { for } i=1,2
\end{gathered}
$$

with

$$
\Phi\left(\dot{\alpha}_{i}\right)=k\left|\dot{\alpha}_{i}\right| \text { and } \mathcal{T}=[-1,1]
$$

Using Theorem 2, we obtain that shakedown occurs if

$$
\epsilon_{T}^{\max } \leq 3 \frac{k}{E}
$$

As is classical in shakedown theory, the results only depend on the extreme values $\left\{0, \epsilon_{T}^{\max }\right\}$ reached by $\epsilon_{T}(t)$ and do not require the knowledge of $\epsilon_{T}(t)$ at each time $t$.

We now investigate the asymptotic behaviour of the three-bar structure when the loading is periodic in time and possibly varies outside of the shakedown domain predicted by (11). We 

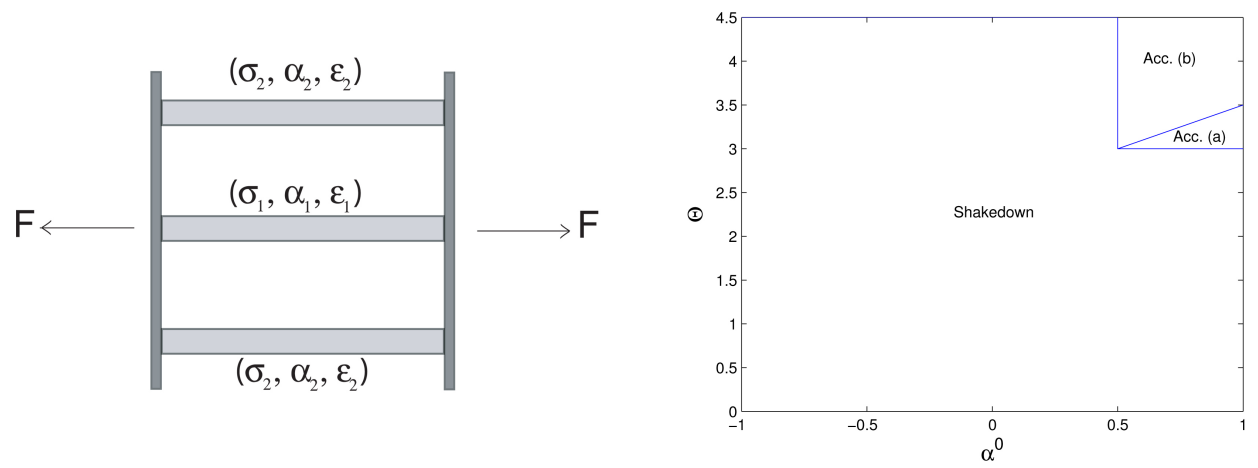

Figure 1: Map of the asymptotic regime (right) for the three-bar problem (left).

limit our attention the case $F=0$ and consider the cyclic loading $\epsilon_{T}(t)$ of period $T$ defined on $[0, T]$ by

$$
\epsilon_{T}(t)= \begin{cases}\frac{2 t}{T} \epsilon_{T}^{\max } & \text { for } 0 \leq t \leq T / 2 \\ 2\left(1-\frac{t}{T}\right) \epsilon_{T}^{\max } & \text { for } T / 2 \leq t \leq T\end{cases}
$$

That evolution problem can be solved explicitly [24]. For all values of the loading parameter $\Theta=\epsilon_{T}^{\max } E / k, \alpha_{1}(t)$ converges towards a cyclic solution. More precisely, for $0 \leq \Theta \leq 3$, shakedown occurs for all value of $\alpha^{0}$, as expected from (11). For $3 \leq \Theta \leq 9 / 2$, the behaviour of the system is more complex than its counterpart in plasticity: depending on the initial state $\alpha^{0}$, there is either alternating plasticity or shakedown. In the cases where alternating plasticity occurs, the stabilized cycles corresponding to different initial conditions are not identical, i.e. they do not simply differ by a constant as they do in the plastic case. The asymptotic amplitude of variation of $\alpha_{1}(t)$ is indeed either equal to $(\Theta-3) k / E$ (case (a)) or to $\alpha^{0}-k / 2 E$ (case (b)).

Figure 1(right) gives a map of the asymptotic regime in the plane $\left(\alpha_{0}, \Theta\right)$. We can observe that Theorem 2 gives the largest value of $\Theta$ for which shakedown occurs whatever the initial state is. Outside of the domain predicted by Theorem 2, the asymptotic regime depends both on the loading and on the initial state, and therefore is not path-independent. A notable consequence is that direct methods used in plasticity should not be transposed without caution to situations where constraints are imposed on the state variables: the path-independence of the asymptotic regime, which is the prerequisite of such methods, is no longer true in the presence of constraints.

\section{Concluding remarks}

In this paper have been presented some shakedown theorems in non-smooth mechanics, applying to situations where constraints are imposed on the internal variables. Those theorems take the form of sufficient conditions ensuring that the energy dissipation remains bounded in time, independently on the initial state. When the loading exceeds the shakedown limits provided by those theorems, the asymptotic regime is generally dependent both on the loading and on the initial state of the structure. That feature is not found in plasticity and stems directly from the presence of constraints on the internal variables. This notably shows that adding constraints on the internal variables has a profound impact on the properties of the structural problem, and therefore should be analyzed with care. 


\section{References}

[1] W. T. Koiter, General problems for elastic solids, Progress in solid mechanics, 1960.

[2] E. Melan, Theorie statisch unbestimmter Systeme aus ideal-plastischen Baustoff, Sitz.Berl.Ak.Wiss. 145 (1936) $195-218$.

[3] B. Halphen, Accommodation et adaptation des structures élastoviscoplastiques et plastiques, Association amicale des ingénieurs anciens élèves de l’ENPC, 1978.

[4] E. Wesfreid, Etude du comportement asymptotique pour un modèle viscoplastique, C.R.Acad.Sci.Paris A 290 (1980) 297-300.

[5] J. Zarka, J. Frelat, G. Inglebert, A New Approach to Inelastic Analysis of Structures, Martinus Nijhoff Publishers, Dordrecht, 1988

[6] S. Akel, Q. Nguyen, Determination of the cyclic response in cyclic plasticity, In: Owen, D.R.J. et al. (Eds), Computational Plasticity: Models, Software and Applications, Pineridge Press, Swansea, 1989.

[7] M. Peigney, C. Stolz, Approche par contrôle optimal des structures élastoviscoplastiques sous chargement cyclique, C.R.Acad.Sci. Paris, Série II 329 (2001) 643-648.

[8] M. Peigney, C. Stolz, An optimal control approach to the analysis of inelastic structures under cyclic loading, J.Mech.Phys.Solids 51 (2003) 575-605.

[9] H. Maitournam, B. Pommier, J. J. Thomas, Détermination de la réponse asymptotique d'une structure anélastique sous chargement cyclique, C.R.Mecanique 330 (2002) 703-708.

[10] B. Halphen, Q. S. Nguyen, Sur les matériaux standards généralisés, J.Mécanique 14 (1975) 1-37.

[11] D. Pham, On shakedown theory for elastic-plastic materials and extensions, J.Mech.Phys.Solids 56 (2008) 19051915.

[12] X. Feng, Q. Sun, Shakedown analysis of shape memory alloy structures, Int J. Plasticity 23 (2007) 183-206.

[13] S. Govindjee, C. Miehe, A multi-variant martensitic phase transformation model: formulation and numerical implementation, Comput. Methods Appl. Mech. Engrg. 191 (2001) 215-238.

[14] M. Peigney, J. Seguin, E. Hervé-Luanco, Numerical simulation of shape memory alloys structures using interiorpoint methods, Int. J. Sol. Struct. 48 (2011) 2791-2799.

[15] M. Frémond, Non-smooth thermomechanics, Springer, 2002.

[16] M. Kružík, A. Mielke, T. Roubícek, Modelling of microstructure and its evolution in shape-memory alloy single crystals, in particular in CuAlNi, Meccanica 40 (2005) 389-418.

[17] H. Brézis, Opérateurs maximaux monotones et semigroupes de contractions dans les espaces de Hilbert, NorthHolland, Amsterdam, 1972.

[18] A. Souza, E. Mamiya, N. Zouain, Three-dimensional model for solids undergoing stress-induced phase transformations, Eur.J.Mech. A 17 (1998) 789-806.

[19] F. Auricchio, L. Petrini, A three-dimensional model describing stress-temperature induced solid phase transformations: solution algorithm and boundary value problems, Int.J.Num.Meth.Eng. 61 (2004) 807-836.

[20] K. Hackl, R. Heinen, An upper bound to the free energy of $n$-variant polycrystalline shape memory alloys, J.Mech.Phys.Solids 56 (2008) 2832-2843.

[21] M. Peigney, A non-convex lower bound on the effective free energy of polycrystalline shape memory alloys, J.Mech.Phys.Solids 57 (2009) 970-986.

[22] M. Peigney, On the energy-minimizing strains in martensitic microstructures-Part 1: Geometrically nonlinear theory, J.Mech.Phys.Solids 61 (2013) 1489-1510.

[23] M. Peigney, On the energy-minimizing strains in martensitic microstructures-Part 2: Geometrically linear theory, J.Mech.Phys.Solids 61 (2013) 1511-1530.

[24] M. Peigney, Shakedown theorems and asymptotic behaviour of solids in nonsmooth mechanics, Eur. J. Mech. A 29 (2010) 785793

[25] Q. S. Nguyen, On shakedown analysis in hardening plasticity, J.Mech.Phys.Solids 51 (2003) 101-125.

[26] J. Mandel, B. Halphen, J. Zarka, Adaptation d'une structure élastoplastique à écrouissage cinématique, Mech. Res. Comm. 4 (1977) 309-314. 